\title{
Kernprobleme mit der Seele
}

\section{Wie bildet sich die Seele aus?}

Unsere zweijährige Tochter spielt gerne mit einer Babuschka. Offensichtlich faszinieren sie die verschachtelten Puppen. Mit ihren kleinen Händen arbeitet sie sich von Hülle zu Hülle bis zum harten Kern vor. Die feinmotorische Meisterleistung wird vom Vater gebührend bewundert. Ihn beeindruckt auch, dass die einzelnen Hüllen Namen bekommen. Die äusserste Hülle heisst Mama. Sie umhüllt alles. Wie sinnig! Mama war - als die Namensgebung erfolgte gerade schwanger. Die nächste Schale trägt den Namen Papa. Ich will das nicht interpretieren, weise aber darauf hin, dass die $\mathrm{Ba}-$ buschka eigentlich Mamuschka heisst. Dann folgen Flurina und schliesslich Anna Lea, der Name der kleinen Schwester, die noch nicht geboren ist. Alle Hüllen sehen zum Verwechseln ähnlich aus. Sie unterscheiden sich nur durch ihre Grösse. In letzter Zeit hat das Spiel mit den verschachtelten Puppenhüllen allerdings seinen Reiz verloren. Ein Grund könnte sein, dass Flurinas Versuche, auch den harten Kern zu schälen, ständig am Widerstand der Materie scheiterten. Es ging nicht weiter. Die Mamuschka gab keinen Blick in ihr Innerstes frei. Flurina hatte ein Kernproblem.

Wollte meine Tochter die Seele der Puppe ergründen? Das ist kaum anzunehmen. Flurina macht sehr wohl einen Unterschied zwischen Spielzeug und Lebewesen. Mit den Puppen werden Experimente durchgeführt. Man kann die Mamuschka zum Beispiel mit Sand oder Legosteinen füllen oder auf den Boden fallen lassen. Ihre kleine Schwester, die in der Zwischenzeit auf die Welt kam, würde Flurina niemals in dieser Weise traktieren. Unterscheidet sie instinktiv? Sie hat natürlich eine Ahnung vom Beseelten. Flurina verfügt andererseits noch nicht über die Kapazitäten, diese Unterscheidung kognitiv zu erfassen, geschweige denn sprachlich $\mathrm{zu}$ formulieren. Dinge können in der Vorstellungswelt einer Zweijährigen ja durchaus noch »beseelt" sein. Bestimmtes Spielzeug hat eine eigenartige Zwischenstellung zwischen Ding und Lebewesen. Im Leben von Flurina hat denn auch "Mige«, ihre Stoffmaus, eine zentrale Bedeutung. Sie ist Trösterin, Schmusepartnerin und Bettgefährtin. (Das hindert Flurina nicht daran, sie hin und wieder unzimperlich zu drangsalieren. "Mige« hat ein entsprechend lädiertes Äusseres.) Es gibt einen Übergangsbereich, in dem die Unterscheidung zwischen Beseeltem und Unbeseeltem geübt wird. Übergangsobjekte, wie Mige, vermitteln zwischen dem Ich des Kindes, das sich von seiner 
Mamuschka ablöst und der verschachtelten Welt, die entdeckt sein will.

Für die Erforschung der geistigen und seelischen Entwicklung des Menschen haben Beobachtungen des kindlichen Umgangs mit Übergangsobjekten wichtige Impulse gegeben. Wer versteht, wie sich die kindliche Seele entwickelt, gewinnt auch Einblicke in das Innenleben eines reifen Erwachsenen oder ein Verständnis für seine Fehlentwicklungen. Wenn beispielsweise Erwachsene ihre Spielzeuge behandeln als seien es beseelte Fetische oder mit lebendigen Menschen umgehen, als seien diese Spielzeuge, ist etwas schief gelaufen in ihrer Entwicklung. Menschliches Leben lässt sich so gesehen als eine zunehmende Verhüllung, Verschichtung und Verdichtung von retwas` verstehen, das in der Anfangsphase noch durchsichtiger ist und sensibel auf Störungen reagiert. Denn was sich entwikkeln muss, ist verwundbar. Unter Umständen kann der Verhüllungs,Verschichtungs- und Verdichtungsvorgang zu Verhärtungen führen. Das Erwachsenen-Ich bildet dann eine harte Schale, vielleicht sogar einen Panzer, der das Ich des Kindes erstickt. Es kann auch das andere geschehen. Das Erwachsenen-Ich hat sich nicht richtig entwikkelt und schützt den empfindlichen und empfänglichen Kern zu wenig. Für die Reifung eines Menschen ist beides entscheidend: die Abdichtung schützender Innenschichten und das Durchlässigbleiben zum lebendigen Kern.

\section{Was bildet Seele ab?}

Weil wir nicht recht wissen, wie wir "es" nennen sollen - Ich? Selbst? Geist? Gemüt? - nennen wir es Seele. Um diese Seele etwas besser zu verstehen, möchte ich noch einmal auf die Kerneinsicht des "Mamuschka-Modells" zurück kommen. Die innerste Einheit, die der ganzen Puppe ihre Form und Identität verleiht, ist im Kern verborgen.

Ich rede in Bildern, weil die Beschreibung seelischer Vorgänge ins Innerste führt. Oder wäre es angebrachter zu sagen, zum Innersten? Ist die Seele das Innerste, muss der Versuch weiter vorzudringen den harten Kern noch einmal zu enthüllen! -, am Widerstand der Materie scheitern. Meine Tochter, behauptete ich, hat eine Vorstellung davon, was beseelt und was unbeseelt ist. Sie kennt auch Dinge, die phasenweise bewohnt sind. Diese Vorstellungen gewinnen mit zunehmendem Alter an Differenziertheit und Konturiertheit. Aber die Einsicht hat ein natürliches Ende. Es geht nicht weiter. Und das, was nicht abbildbar ist, wird als das Zeichen "Seele« ins Bild gerückt und auf den Begriff gebracht. Was dem Auge verborgen bleibt, kommt als »Kern« zur Anschauung, damit es bezeichnet, verhüllt und 


\section{Ralph Kunz}

so auf den Begriff gebracht werden kann. Zur Vorstellung der Seele gehört die primitive und höchst elementare Vorstellung, dass setwas im Inneren des Menschen wohnt.

Das Kernproblem bleibt also. Das innerste "Etwas" ist im Leib verborgen und offenbart sich nur mittelbar über seine Körperhülle. Es drängt nach aussen; in Gesten, Worten oder anderen Formen der Äusserung. Allgemeiner gesagt: Jede Äusserung stammt ursprünglich aus einem Innenraum und wird über einen Zwischenraum durch Zeichenträger in einen anderen Innenraum vermittelt. Seelen wissen von anderen Seelen nur mittels deren Äusserungen. Die Zeichenträger, die gewissermassen als Überbrückungsobjekte vermitteln, sind körperlich, auch wenn sie nur gedacht sind. Zeichenkörper bilden einen Übergangsbereich, einen Raum, in dem und durch den Lebewesen überhaupt kommunizieren können. Es gibt keine Signifikation ohne Signifikanten. Diese können zum Spielzeug werden, ein Eigenleben gewinnen, neue Signifikationsprozesse auslösen.

Trifft dies zu, können Seelen nur indirekt und mittelbar kommunizieren. Sie brauchen einen symbolischen Übergangsbereich. Sie spielen, wenn sie einander begegnen, miteinander das Spiel der Kommunikation. Die Körpermetaphorik ist deshalb eine heimliche und zugleich unheimliche Konstante für die Vorstellung unserer inneren Welt. Wir sind beseelte Leiber und können uns Seelen nur verkörpert vorstellen. Das epistemologische Problem, das uns unsere abgeschlossene Körperlichkeit beschert, entlarvt jeden Versuch, sich um die Seele eines anderen zu sorgen, als höchst schwierige Divinationsaufgabe. Wie kommen wir denn zum Kern eines anderen, wenn wir doch nur Hülle erkennen? Alles, was wir wahrnehmen, ist eingeschlossen in eine Zeichenhülle. Wir erkennen Menschen nur mittels der Zeichen, die sie herausgeben. Um den Kern der Botschaft zu enthüllen, sind wir wiederum auf ein Zeichenrepertoire einen Code - verwiesen.

Noch schwindelerregender ist die Introspektion - eine Art Selbstlektüre der Zeichen, die wir sind. Wie sollen wir selbst unsere Seele erkennen und verstehen? Wie gelangen wir zu unserem Innersten? Das ist eine Anstrengung, die immer prekär bleibt, auch dann, wenn wir im Umgang mit anderen und uns selbst lernen, uns in einer Art Selbstsemiose zu üben, d.h. uns besser zu lesen oder, im Bild gesprochen, unterschiedliche Schichten $\mathrm{zu}$ bezeichnen und so scheinbar - ins eigene Innere vorzudringen. Denn wir müssen, auch wenn wir uns selbst lesen, einen Zwischentext verfassen. Die Seele, das innerste Stück, bleibt terra incognita, ein innerer Raum unter der Oberfläche des Körperzeichens, zu dem wir keinen anderen Zugang kennen und aus dem heraus wir keinen andern Ausweg wissen, als 
erneut das Zeichen zu setzen, das sich als lebendiges, sich selbst setzendes Zeichen auszeichnet.

\section{Was zeichnet Seele aus?}

Es ist nicht weiter schwierig, den Versuch, hinter den Bildern unserer Vorstellung eine reale Substanz auszumachen und einen Wesenskern zu erkennen, als Illusion zu entlarven. Nur sind wir bei diesem Versuch erst recht wieder im Spiel. Auch in der semiotischen Enthüllung der Bilder kommt letztlich nichts anderes als das Zeichenmaterial unserer verinnerlichten Welt- und Selbstbilder ins Spiel. Es kann nichts anderes zum Vorschein kommen. Auch die Seele ist "nur« ein Zeichen. Das ist sicherlich wahr, es wäre aber wenig ratsam, die Enthüllung dieser Enthüllung als Einsicht hinter die Kulissen zu bezeichnen. Wo kämen wir da hin?

So ist auch die Enthüllung der Enthüllung eine Verhüllung mit Zeichen. Man kann das Kernproblem auch produktiver und konstruktiver formulieren. Dass die Seele sich, wie durch spiegelndes Glas, selbst bezeichnen kann, zeichnet sie als Teil unserer Existenz aus und versetzt uns überhaupt in die Lage, ein echtes Selbstgespräch zu führen, das wir nicht als Selbsttäuschung bezeichnen. (Wenn wir dies täten, müssten wir zumindest die Feststellung, dass wir uns selbst täuschen, als authentisches Selbstgespräch bezeichnen.) Über die Existenz der Seele gibt es zugegebenermassen trotzdem keine verlässlichen Aussagen. Es gibt andererseits auch keinen vernünftigen Grund, diese zu bestreiten. Es ist ja nicht auszuschliessen, dass uns aus unserem Inneren Zeichen erreichen, die wir nicht selbst und selbstbewusst gesetzt haben, nur weil wir diese qua unserer beschränkten kommunikativen Möglichkeiten mittels einer Selbstunterscheidung und durch einen inneren Dialog ins Bewusstsein heben können. Jacques Monod verwendet in seinen philosophischen Überlegungen zur modernen Biologie der Seele deshalb die Formel von der Selbstbezeugung. Weil ich ihn für einen unverdächtigen Zeugen der selbstbezeugten Existenz der Seele halte, sei er hier zitiert: "Verzichten wir auf die Illusion, in der Seele eine immaterielle 'Substanz` zu sehen, dann leugnen wir nicht deren Existenz, sondern wir beginnen im Gegenteil, die Komplexität ... zu erkennen, die zusammen das Wesen ausmachen, das sich in uns einmalig und unwiderleglich selber bezeugt«.

Es versteht sich von selbst, dass der Komplexität unseres verschachtelten Selbst mit dem primitiven Mamuschka-Modell nicht beizukommen ist. Die Grenzen dieserVorstellung sind zu offensichtlich. Unbeantwortet bleibt die Frage, wie der Geist in Fleisch und Blut kommt. Wo, in welcher Schicht, sitzt eigentlich die Seele? Hat 


\section{Ralph Kunz}

sie eine Haut? Und wenn wir unsere Selbstgespräche abhören: Wie unterscheiden wir die Stimme unseres Gewissens von den Stimmen, die in uns flüstern? Wie kommt es, dass zwei Seelen in unserer Brust miteinander streiten können? Wie geht es der Seele, wenn der Verstand gegen das Gefühl spricht? Ich finde das Bild von ineinander verschachtelten und einander entsprechenden, miteinander sprechenden, selbstreflektierenden und durchsichtigen Ich-Hüllen dennoch attraktiv. Es hilft, oberflächliche und tiefere Schichten im Innenleben zu unterscheiden. Zur tiefsten Schicht gehört jene selbstbezeugende Stimme, die, gerade weil sie selbstbezengend ist, uns aus der Selbstabgeschlossenheit herausruft und zugleich einen Anderen anruft, der sich selbst als Zeichengeber bezeugt. Zugegeben, der Schluss von der eigenen Selbstbezeugung auf diese andere Existenz ist nur kontingent und nicht notwendig. Mehr lässt sich nicht sagen, mehr Gewissheit nicht erreichen. Das gehört zum Spiel! Aber im Selbstgespräch der Seele, das keines sein will, weil es die Selbstbezeugung des anderen vernehmen will, drückt sich Vertrauen aus, dass der andere zuhört. Das muss eine "Seele von einem Mensch» sein!

\section{Wie soll ich Dich empfangen und wie begegn ich Dir}

Nennen wir dieses Selbstgespräch Gebet, nennen wird diese "Seele eines Menschen" gottgleich. Wenn nämlich diese Seele eines Menschen Gott bezeichnet und Gott für diese Seele zeugt, kennt sie nicht nur alle Zeichen von A bis Z, sie kann auch lesen, hören, empfangen und verstehen. Und weil sie empfänglich ist, sendet sie auch. Wo und wie vernehmen wir die viva vox, die uns mitteilt, dass unsere Seele mit einer göttlichen Seele verbunden und Gottes Seele für Menschen empfänglich ist? Wir begegnen ihr mit und durch unsere Empfänglichkeit für Gott. Und wo begegnen sich die göttliche Empfänglichkeit für den Menschen und die menschliche Empfänglichkeit für Gott? In einem Zeichen, das Gott setzt, in einem Wort, das auf uns hört und sich uns zuspricht. Wenn Gott diese Seele ist, auf die wir hören, wenn wir mit uns sprechen, können wir sie nur in Zeichengestalt wahrnehmen. Weil sich Gott uns als der Andere, aber nicht in konkret physischer Gestalt offenbart, sind wir auf Zeichen angewiesen, die die Seele eines Menschen, die Gottes Selbstbezeugung bezeugt, hinterlassen hat.

Die Überlieferung dieser Hinterlassenschaft ist in der Schrift gespeichert als Zeugnis von Christus Jesus. Aber der Geist Jesu versteckt sich nicht im Buchstaben. Wir hätten sonst ein Kernproblem. Es liest, spricht und zeugt der Geist für sich selbst durch seine Zeichenträger. Das kann in Geschichten, Liedern oder Gebeten sein. Es sind auf jeden Fall lebendige Menschen, die Christus durch 
inspirierte Interpretation der Symbole ihre Stimme leihen. Monod möge es mir verzeihen, wenn ich sein Bekenntnis zur Existenz der Seele auf diese Weise auslege. Aber mir scheint es evident, dass wir mit dem Abschied von der Illusion, in Gott eine immaterielle sSubstanz zu sehen, nicht seine Existenz leugnen, sondern im Gegenteil beginnen, die Komplexität der Zeichenprozesse zu erkennen, die zwischen den Schichten unserer Existenz das Wesen ausmachen, das sich in uns einmalig und unwiderleglich selber bezeugt.

Was in dieser eigenartigen Figur des inneren Dialogs mit dem Anderen, das in mir wohnt, tatsächlich steckt, entzieht sich meinem Zugriff. Versuche, Gott auf den Begriff und mich selbst völlig in Griff zu bekommen, werden am Widerstand der Materie scheitern. Von Gott hingegen wird bezeugt, dass er im Verborgenen sieht (Mt $6,4)$ und kein Geschöpf vor ihm unsichtbar ist (Heb 4,13). Wohin sollen wir fliehen vor dem, der unsere Seelen kannte, als er im Mutterschoss gewoben hat? (Ps 139,13f.) Das wäre eine beunruhigende Frage, wenn nicht auch die Sorge Gottes um unsere Seele bezeugt würde. Wir sind kein Spielzeug des Schöpfers. Gott hat uns nicht im Griff.

Wenn wir das begriffen haben, können wir beten, wenn wir beten, begreifen wir etwas davon. Eines der schönsten Gebete, das ich kenne, ist ein Mamuschka-Gebet. Mit ihm will ich diesen Annäherungsversuch an das Seelische beschliessen. Im 131. Psalm heisst es mit kleinen Variationen meinerseits:

»Herr,

mein Herz ist nicht hoffärtig

und meine Augen sind nicht stolz;

ich gehe nicht mit Dingen um,

die mir zu tief im Innern

und zu wunderbar sind.

Fürwahr, ich habe meine Seele gestillt und beruhigt.

Wie ein Entwöhnter ist stille in mir meine Seele.

Harre, Israel,

auf den Herrn von nun an bis in Ewigkeit». 